\title{
Isolation of attosecond pulses from the attosecond lighthouse
}

\author{
TJ Hammond ${ }^{1,2,5}$, Emeric Balogh ${ }^{3}$ and Kyung Taec Kim ${ }^{3,4}$ \\ ${ }^{1}$ University of Ottawa and National Research Council Canada, 100 Sussex Dr, Ottawa, ON K1A 0R6, \\ Canada \\ ${ }^{2}$ Institute for the Frontier of Attosecond Science and Technology, CREOL and Department of Physics, \\ University of Central Florida, Orlando, FL 32816, USA \\ ${ }^{3}$ Center for Relativistic Laser Science, Institute for Basic Science, Gwangju 61005, Korea \\ ${ }^{4}$ Department of Physics and Photon Science, Gwangju Institute of Science and Technology, Gwangju \\ 61005, Korea
}

E-mail: thammond@uottawa.ca

Received 31 July 2016, revised 13 September 2016

Accepted for publication 26 September 2016

Published 16 December 2016

\begin{abstract}
This paper proposes and verifies methods to improve the isolation of the attosecond pulses generated via the attosecond lighthouse technique in gases. We find that the converging fundamental field can compensate for the inherent diverging wavefront of the attosecond pulses, but is only valid over a limited portion of the spectrum and sacrifices the maximum attainable photon energy. We discuss two methods of manipulating the fundamental field to control the divergence of the attosecond pulses: the first method uses a perturbing beam that manipulates the wavefront, and the second method controls the fundamental dispersion. These methods improve the isolation of the attosecond pulses up to the cutoff photon energy.
\end{abstract}

Keywords: high-order harmonic generation, attosecond pulse generation, nonlinear optics

(Some figures may appear in colour only in the online journal)

\section{Introduction}

Attosecond pulse trains are generated routinely in the lab via high harmonic generation $[1,2]$. When a laser pulse with intensity $10^{14}-10^{15} \mathrm{~W} \mathrm{~cm}^{-2}$ is incident on a gas, an electron in the gas can be separated from its parent atom or molecule, become accelerated in the oscillating laser field, and recollide with the parent ion up to a maximum energy of $E_{h \nu}^{\max }=V_{p}+3.17 U_{p}$, where $V_{p}$ is the ionization potential of the gas, and $U_{p}$ is the ponderomotive energy of the field [3]. Upon recollision, the electron releases a burst of coherent radiation in the form of extreme ultraviolet radiation (XUV) [4]. This process occurs every half cycle, leading to a series of high-order odd harmonics of the fundamental laser field [5]. The single atom response to the fundamental field can be

\footnotetext{
5 This article belongs to the special issue: emerging leaders, which features invited work from the best early-career researchers working within the scope of JPhysB. This project is part of the Journal of Physics series' 50th anniversary celebrations in 2017. T J Hammond was selected by the Editorial Board of JPhysB as an Emerging Leader.
}

controlled to generate a single isolated attosecond pulse through the manipulation of polarization $[6,7]$, the pulse duration $[8,9]$, judicious choosing of the nonlinear medium $[10,11]$, or a combination of these techniques $[12,13]$ to gate the interaction of the fundamental laser field with the nonlinear medium. Adding the second harmonic of the fundamental field relaxes the stringent pulse duration requirements [14-17]. It is also possible to manipulate the macroscopic response by directing the sequential pulses uniquely in the far field to achieve isolation post-creation [18]. This method is the principle behind the attosecond lighthouse.

The attosecond lighthouse is a technique that rapidly rotates the wavefront at the focus of an intense beam $[19,20]$. The ultrafast wavefront rotation creates a series of spatially separated attosecond pulses and acts as a beamsplitter at the time of generation, uniquely directing each attosecond pulse to the far field [21-23]. It is possible to generate more than the necessary number of pulses for a pump-probe experiment, while using the remaining pulses to monitor the XUV flux and fundamental field character (which can drift over the 


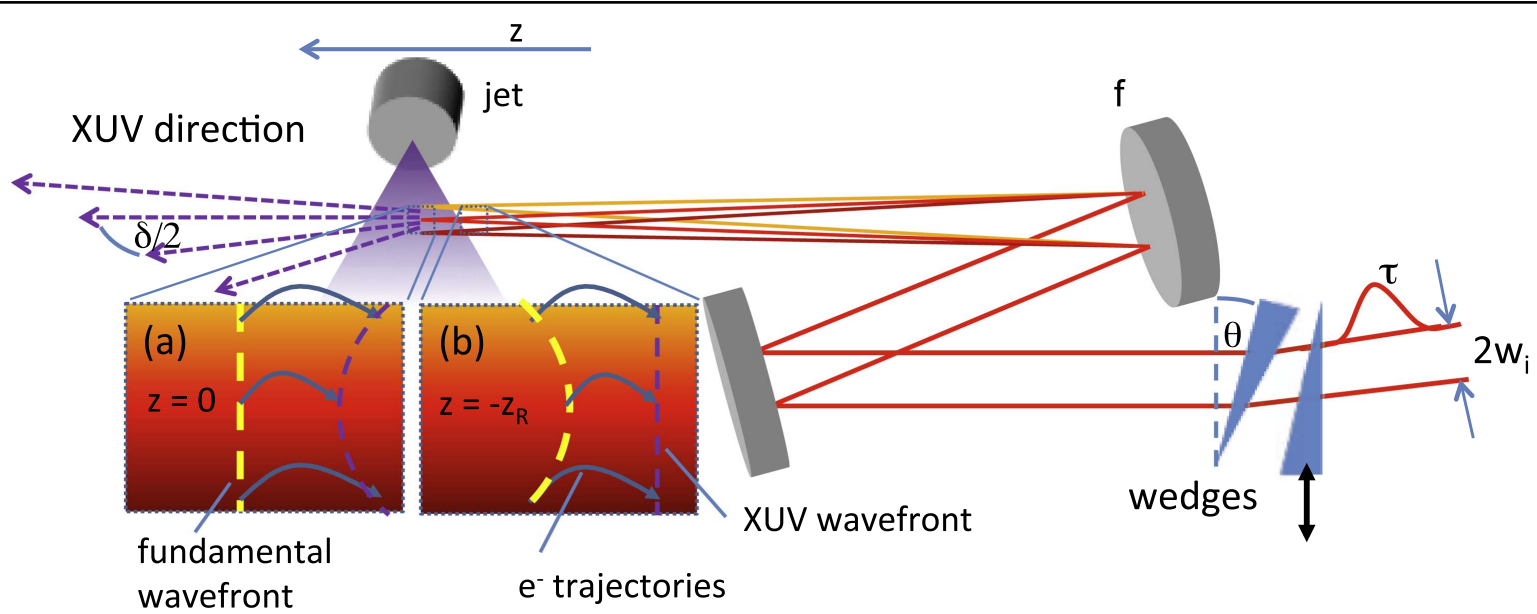

Figure 1. Experimental setup of the attosecond lighthouse. The misaligned wedges, relative angle $\theta$, tilt the pulse front causing a spatial chirp at the focus; the parallel wedge controls dispersion. The XUV pulses generated in subsequent half-cycles of the fundamental field propagate in unique directions, separated by $\delta / 2$, to a 2D spectrometer and MCP (not shown). Inset: the electron trajectories for a given harmonic order are dependent on the local intensity. (a) At the focus, $z=0$, the fundamental wavefront is flat leading to a diverging XUV wavefront, and (b) before the focus, $z=-z_{R}$, the fundamental wavefront is converging and compensates for the inherent diverging wavefront of the attosecond pulse.

duration of an experiment) [24]. Furthermore, there is no polarization ambiguity with the generated pulses [25, 26], ensuring parallel polarization across the spectrum. Alongside these benefits, this paper will discuss the limitations of the attosecond lighthouse technique, such as decreased cutoff and limited bandwidth isolation, as well as methods to overcome these limitations.

\section{Theory and simulations}

The experimental setup for the attosecond lighthouse is shown in figure 1. A pair of misaligned wedges, with relative angle $\theta$, creates a pulse front tilt in the far field, or a spatial chirp when focused in the near field [27]. The spatial chirp, where the short wavelength and the long wavelength portions of the spectrum are spatially separated, creates a wavefront in which each half-cycle of the fundamental field rotates by an angle $\delta / 2$. The XUV propagates to a $2 \mathrm{D}$ spectrometer and micro-channel plate (MCP). When the wavefront rotation exceeds the divergence of the XUV bursts, then the attosecond lighthouse creates a series of isolated attosecond pulses.

The divergence of the attosecond pulses depends on its phase. In gases, there is an additional phase in the high harmonic generation process, referred to as the atomic phase or the dipole phase [28-31]. This dipole phase, $\phi_{\text {dip }}$, depends on the returning electron trajectory, shown in the inset in figure 1 , and on the intensity of the fundamental field $\phi_{\text {dip }}(z, x) \sim-\alpha_{s, l} I(z, x)$, where $\alpha_{s, l}$ is the phase coefficient for the short (s) and long (l) trajectory, and $I(z, x)$ is the position dependent intensity [32]; here, we only take the short trajectory into account. The on-axis portion of the field has the highest intensity and accumulates the least phase for a given recolliding energy. Off-axis, the lower intensity requires a longer trajectory to reach the same recolliding energy. In the left inset (a), the flat wavefront of the fundamental field leads to a diverging wavefront of the harmonic. In the right inset (b), the converging wavefront compensates for the radially dependent dipole phase, leading to a flat generated wavefront; this latter case has a minimum diverging pulse.

In the far field, the misaligned wedges impose a pulse front tilt on the fundamental field [19, 33],

$$
E_{i}(x, t)=E_{0} \exp \left(-\frac{\left(t+\xi x_{i}\right)^{2}}{2 \tau_{i}^{2}}-\frac{x_{i}^{2}}{w_{i}^{2}}\right) \mathrm{e}^{\mathrm{i}\left(\omega_{\mathrm{L}} t+\phi_{0}\right)},
$$

where $E_{0}$ is the peak electric field in the far field, $\Delta t_{\mathrm{FWHM}}=2 \tau_{i} / \sqrt{\ln (2)}$ is the transform limited full width at half maximum (FWHM) pulse duration, $w_{i}$ is the far field beam waist, $\omega_{\mathrm{L}}$ is the fundamental (angular) frequency, and $\phi_{0}$ is the carrier envelope phase (CEP). At a focal length $f$, the pulse front tilt parameter, $\xi$, governs the wavefront rotation rate $\omega_{r}$ at the focus by

$$
\omega_{r}(\xi)=\frac{w_{i}^{2}}{2 f \tau_{i}^{2}} \frac{\xi}{1+\frac{\xi^{2} w_{i}^{2}}{2 \tau_{i}^{2}}} .
$$

The maximum rotation rate, $\omega_{r}^{\max }=w_{i} / 2 \sqrt{2} f \tau_{i}$, occurs when $\xi=\sqrt{2} \tau_{i} / w_{i}$. That is, the maximum wavefront rotation at the focus is only dependent on the initial beam waist, pulse duration, and focal length. The angle of separation of subsequent attosecond pulses is $\delta / 2=\pi \omega_{r} / \omega_{\mathrm{L}}$.

However, the consequence of the pulse front tilt's leading to the spatial separation of the fundamental frequency components at the focus is that both the minimum waist (in the plane of the pulse front tilt), $w_{f}$, and the pulse duration, $\tau_{f}$, are increased by a factor of

$$
w_{f} / w_{0}=\tau_{f} / \tau_{i}=\sqrt{1+\frac{\xi^{2} w_{i}^{2}}{2 \tau_{i}^{2}}},
$$

where $w_{0}$ is the minimum waist when $\xi=0$. The resulting peak intensity is decreased by a factor of two at $\omega_{r}^{\max }$. 

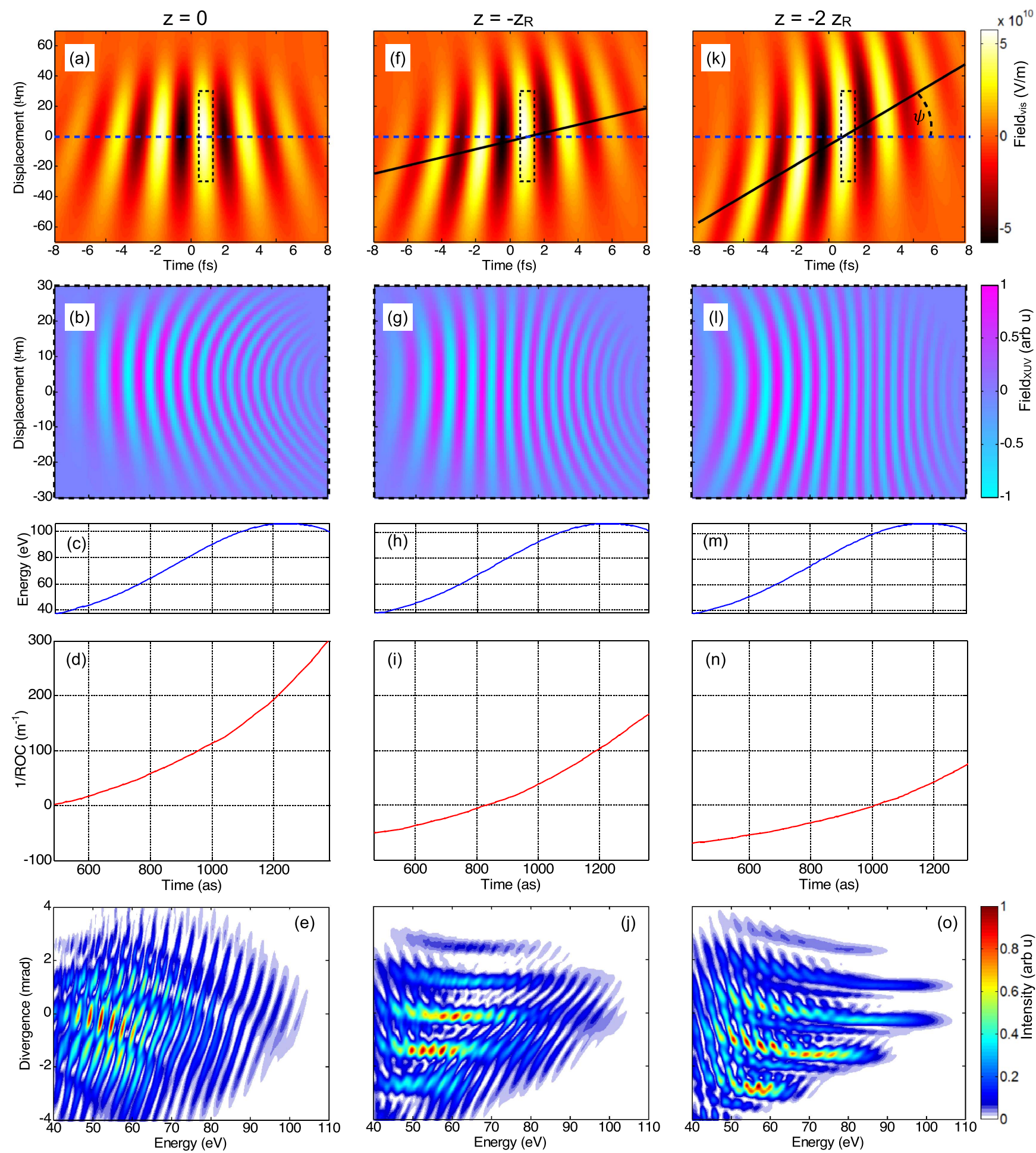

Figure 2. Using the gas jet position to improve isolation ( $z<0$ signifies before the focus). Left column gas jet position $z=0$, middle column $z=-z_{\mathrm{R}}$, right column $z=-2 z_{\mathrm{R}}$. (a), (f), and (k): relative angle, $\psi$, of the propagation axis (blue dash) and the centre-of-mass (black). Top row is the fundamental field with spatial chirp, second row is the magnified view (dashed box) of the induced nonlinear dipole response calculated in SFA of one attosecond pulse, third row is the calculated energy along $x=0 \mathrm{~mm}$ displacement, fourth row is the inverse of the radius of curvature of the XUV wavefront. Rows two-four share a common time axis within one attosecond pulse. The bottom row is the calculated spatially dependent spectrum.

The optimum pulse front tilt for a transform limited pulse has been analytically solved around the focus [33]; here, we numerically calculate the pulse propagation through the focus [34] to allow for perturbing fields and group delay dispersion (GDD).
The effects of the wavefront rotation, the fundamental wavefront curvature on the dipole phase, and the resulting divergence are shown in figure 2 . We calculate dipole response using the strong field approximation (SFA) for a peak intensity of $4.5 \times 10^{14} \mathrm{~W} \mathrm{~cm}^{-2}$ (for all values of $\mathrm{z}$ ), with 

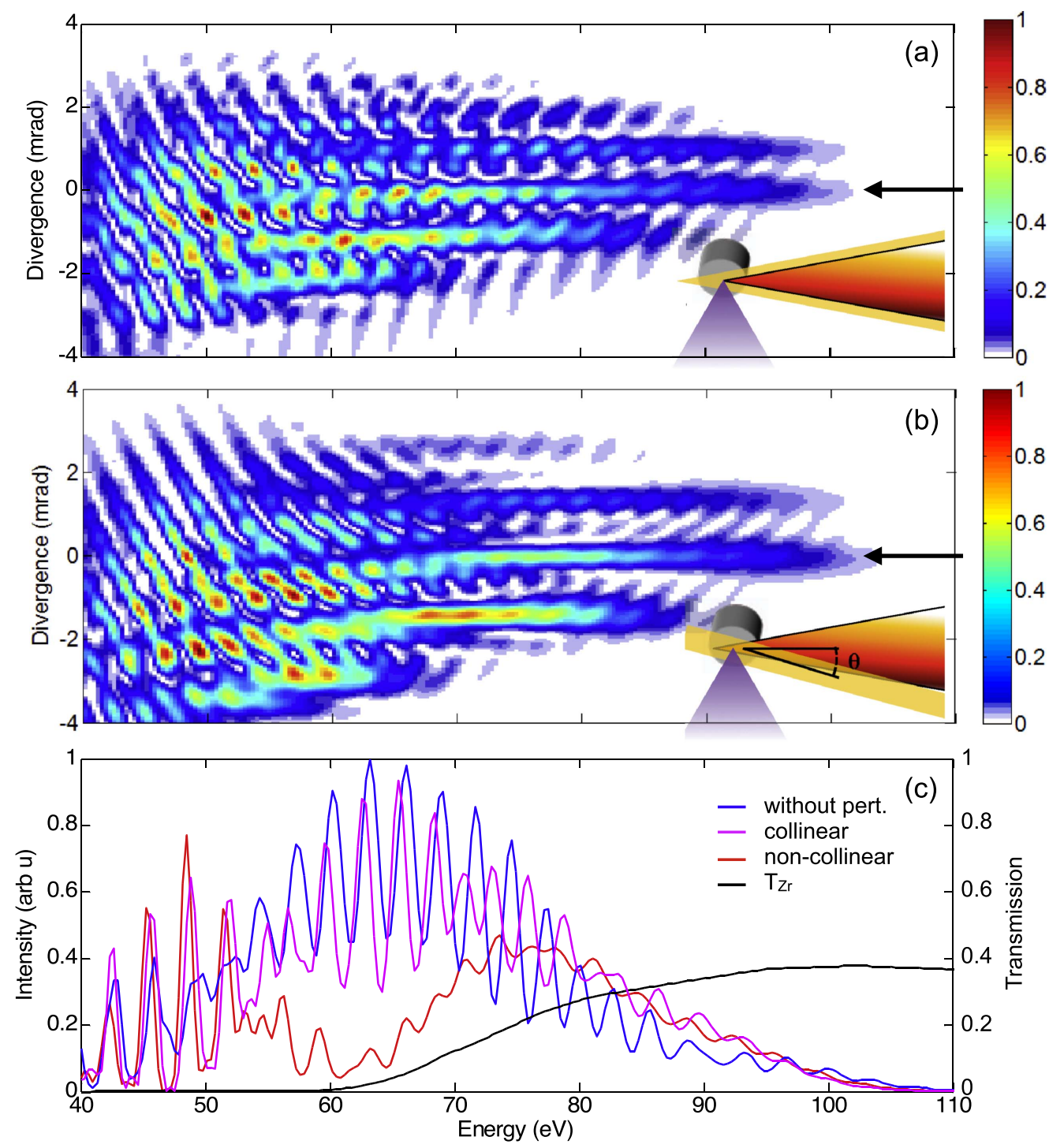

Figure 3. Using a perturbing beam to improve isolation. (a) Collinear setup-inset: the gas jet is placed at $z=0$ and $z_{p}=z_{\mathrm{R}} / 2$. The $I_{p} / I_{0}=4 \% ; \phi_{p}=1.5 \pi$. (b) Non-collinear setup-inset: gas jet at $z=-z_{\mathrm{R}} / 2$, relative angle $\theta=5 \mathrm{mrad}, I_{p} / I_{0}=0.25 \%, \phi_{p}=0.8 \pi$. (c) Lineout along the zero divergence shown with arrow in pink (a) and red (b); also shown is the lineout for $z=-z_{R} / 2$ without a perturbing arm (blue), and the transmission of $300 \mathrm{~nm}$ thick $\mathrm{Zr}$ filter (black).

a fundamental wavelength of $760 \mathrm{~nm}$ and initial pulse duration of $4.5 \mathrm{fs}$. The initial beam parameters are: $w_{i}=2.5 \mathrm{~mm}$, $f=300 \mathrm{~mm}$ mirror (an unchirped minimum waist of $\left.w_{0}=30 \mu \mathrm{m}\right)$, and $\xi=1.5 \mathrm{fs} \mathrm{mm}^{-1}$. The CEP of each column is tuned such that the largest amplitude pulse is along the zero divergence. The left column is the calculation at the focus of the fundamental field $z=0$. The middle column is the calculation when the gas jet is placed one Rayleigh range before the focus $z=-z_{\mathrm{R}}$, where the Rayleigh range $z_{\mathrm{R}}=\pi w_{0}^{2} / \lambda$ and $\lambda=2 \pi c / \omega_{\mathrm{L}}$ is the central wavelength. The right column is for the gas jet placed $z=-2 z_{\mathrm{R}}$ before the focus. The top two rows show the spatio-temporal coupling of the fundamental field (in $\mathrm{V} \mathrm{m}^{-1}$ ) and the dipole response (in arbitrary units), respectively. The second row is the spacetime magnified view of the top row, outlined by the dashed box. The third row is the dipole energy as a function of time along the zero displacement (in $\mathrm{eV}$ ). The fourth row is the inverse of the radius of curvature of the dipole response (in 1/ $\mathrm{m})$; values $<0$ are converging and $>0$ are diverging. Rows two to four share a common time axis over the duration of the highlighted attosecond pulse. The resulting spatially dependent spectra appear in the bottom row, where a temporal filter removes the long trajectory contribution.

At the focus, figure 2(a), the wavefront rotation is apparent, with each fundamental wavefront flat over the focal region. The dipole response (b) shows a flat wavefront for the longer wavelength; the shorter wavelength emitted at a later time has a diverging wavefront (pulses propagate right to left in space). The energy of the generated XUV spans from 40-105 eV (c); the radius of curvature is flat only for the lowest portion of the spectrum (d). The spatially resolved far-field spectrum, (e), has only the lowest portion of the spectrum with resolved pulses.

At $z=-z_{\mathrm{R}}$ (f), the curvature of the fundamental field is optimized to collimate the middle portion of the XUV spectrum. Over the generated photon energy range of $40-80 \mathrm{eV}$, we can see a series of isolated attosecond pulses (j). The modulation 

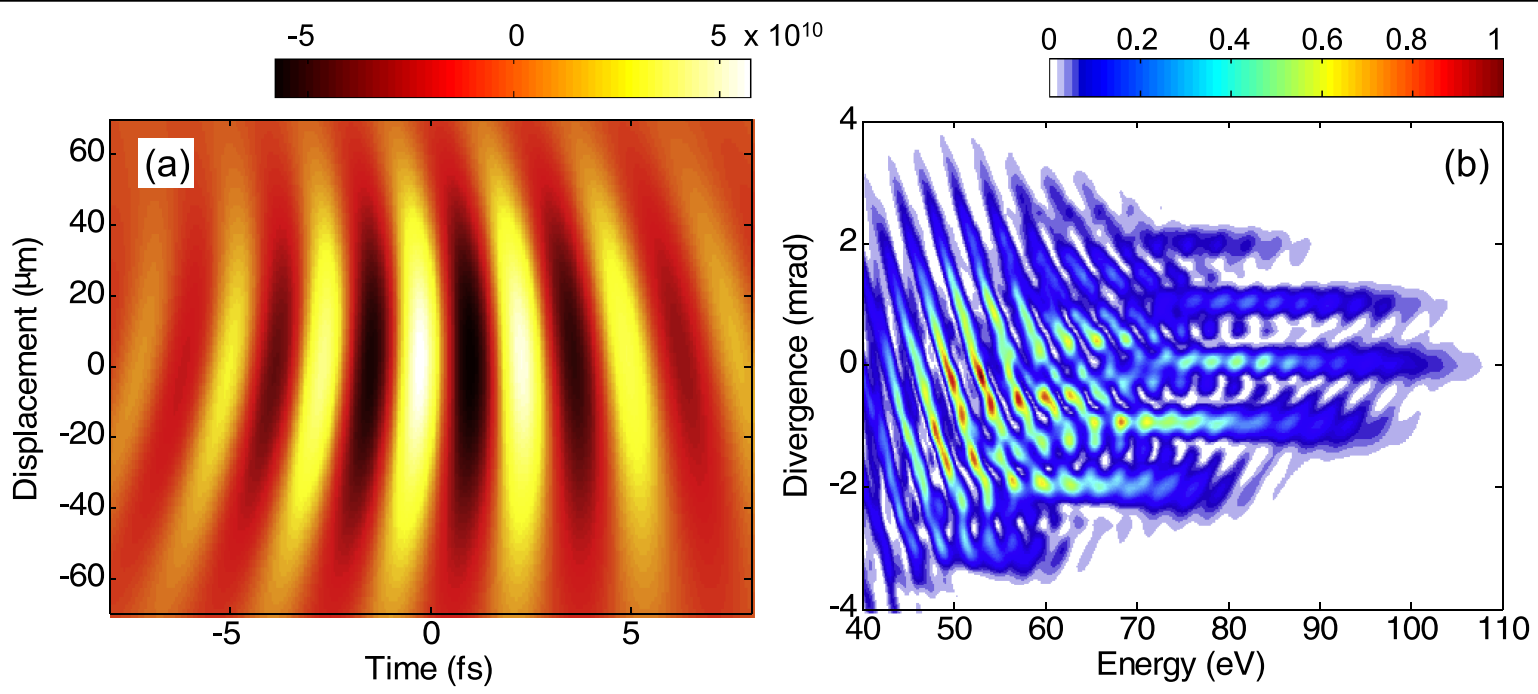

Figure 4. Wavefront control through dispersion. (a) Anomalous dispersion $\left(-6 \mathrm{fs}^{2}\right)$ of the fundamental field modifies the divergence of the attosecond pulses for $z=-z_{\mathrm{R}}$. (b) The resulting spatially resolved spectrum shows clear separation above $75 \mathrm{eV}$.
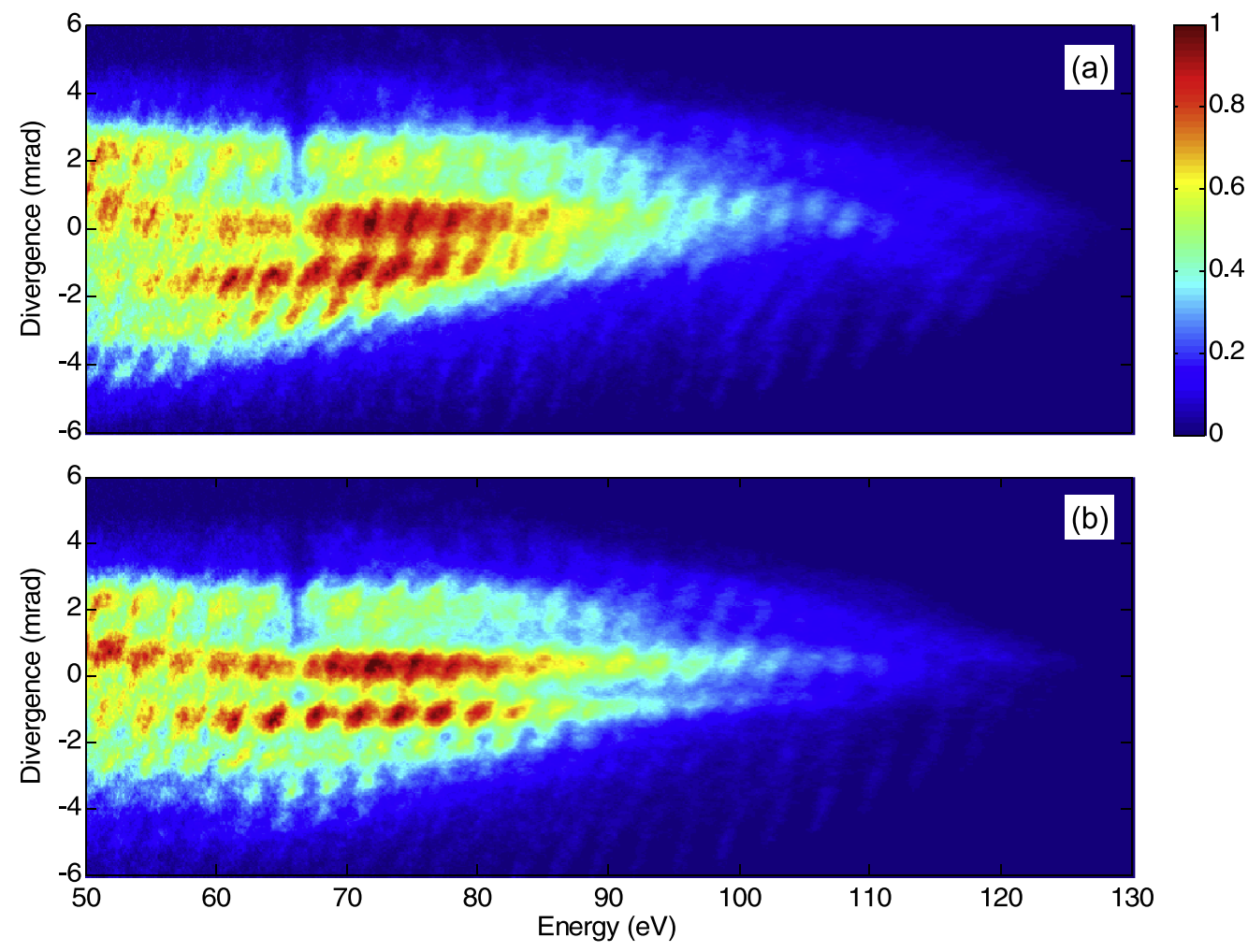

Figure 5. The spatially resolved XUV spectrum from the attosecond lighthouse. (a) Without a perturbing field the maximum photon energy of each attosecond pulse is not well resolved. (b) A non-collinear perturbing arm improves the separation above $80 \mathrm{eV}$.

depth (the fringe visibility) $V=\left(I_{\max }-I_{\min }\right) /\left(I_{\max }+I_{\min }\right)$ is calculated to be $32 \%$ at $80 \mathrm{eV}$, or a pulse contrast $C=4 / V^{2}=40$.

Generating attosecond pulses from $z=-2 z_{\mathrm{R}}(\mathrm{k})$ isolates the attosecond pulses near the maximum photon energy. The spatio-temporal coupling before the focus leads to a walk-off of the centre-of-mass (black solid) and the direction of propagation (blue dash) of the fundamental field, $\psi$. This yaw, $\psi$, originates from the combination of the far field pulse front tilt and the focal spatial chirp, and decreases the rate of wavefront rotation. In spite of this yaw, the fundamental curvature is sufficient to collimate the cutoff energy from $80-110 \mathrm{eV}$ for isolated attosecond pulses.

However, experimentally moving the gas jet to $-z_{\mathrm{R}}$ before the focus decreases the peak intensity by nearly a factor of two, limiting the cutoff harmonic energy. This decrease is in addition to imposing the wavefront rotation that decreases the peak intensity by a factor of two, as mentioned above. The attosecond lighthouse technique alone does not generate high contrast isolated attosecond pulses near the 


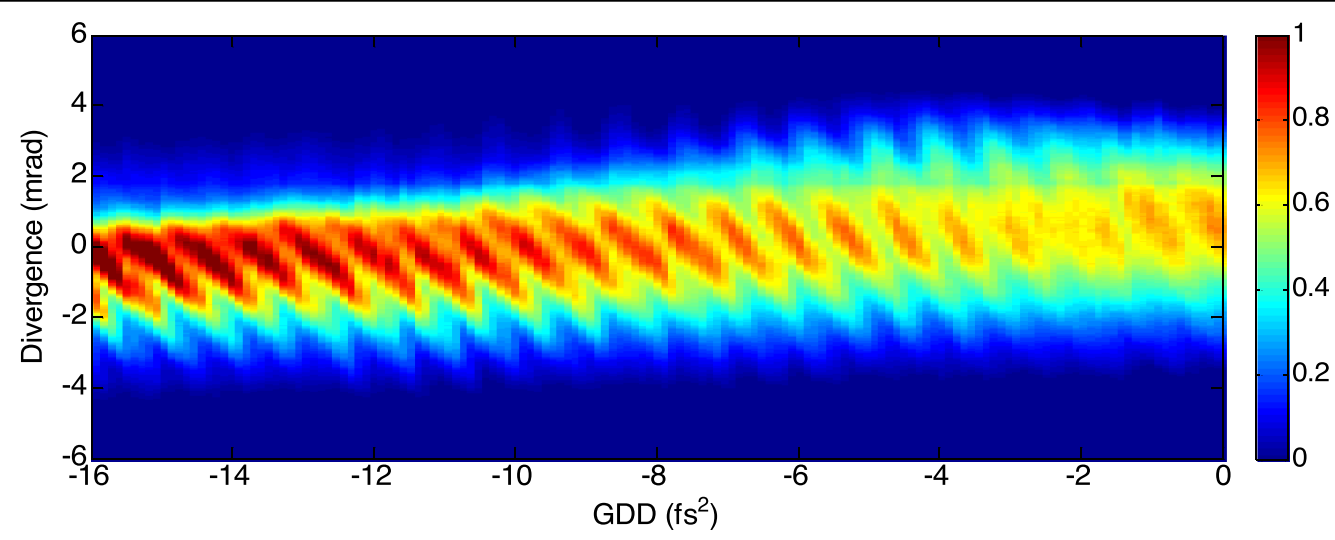

Figure 6. The GDD, tuned through wedge insertion, affects the divergence and spatial separation at $85 \pm 5 \mathrm{eV}$. The optimum separation is at $-5 \mathrm{fs}^{2}$. The wedge also controls the CEP (rapid slope).

maximum available cutoff photon energy. In order to overcome this limitation, we propose two methods to tune the isolated portion of the spectrum. The first is with a perturbing field-collinear and non-collinear-and the second is by tuning the temporal chirp of the driving field.

By introducing a perturbing field, we control the curvature of the driving field near the focus. In this manner, we manipulate the divergence of the generated attosecond pulse while maintaining the high peak intensity required for high harmonic generation. In figure 3(a) we see the effect of a collinear geometry. The inset shows a schematic of the setup, where the gas jet is placed at the focus of the fundamental field (red shaded) $z=0$, while the perturbing arm focus (light orange) is at $z_{p}=z_{\mathrm{R}} / 2$. This setup is similar to that of an alloptical Fresnel zone plate [35]. The converging perturbing field modifies the flat wavefront of the fundamental field at the focus; the resulting total field curvature is converging and decreases the divergence of the generated attosecond pulses. The relative perturbing intensity is $I_{p} / I_{0}=4 \%$, and the relative phase of the two fields is $\phi_{p}=1.5 \pi$. The perturbing field wavelength and beam waist is the same as the fundamental. The perturbing arm can significantly change the cutoff harmonic depending on the relative phase; experimental realisation of this technique would require an interferometrically stable system. Comparing this spectrum to figure 2(e), we see significant improvement on the isolation of the pulses at the cutoff energy.

In a non-collinear setup, shown in figure 3(b), we introduce a plane wave perturbing arm (light orange rectangle) of the same wavelength as the generating field, at a small angle $\theta=5 \mathrm{mrad}$, with relative on-axis intensity $I_{p} / I_{0}=0.25 \%$ and $\phi_{p}=0.8 \pi ; z=-z_{\mathrm{R}} / 2$. The perturbing field (although weak on-axis) is sufficiently strong to modify the curvature of the generating field at $\pm w_{0}$. The modified curvature of the off-axis field decreases the divergence over a small portion of the generated spectrum. Here, the perturbing field isolates the spectrum above $65 \mathrm{eV}$. The isolated portion of the spectrum depends on the relative angle $\theta$, where increasing $\theta$ increases the isolated photon energy.

The lineout along the zero divergence for the different cases (black arrow) is shown in figure 3(c). The spectrum calculated with the gas jet placed $z=-z_{\mathrm{R}} / 2$, unperturbed (blue); at $80 \mathrm{eV}, V=32.3 \%$, or an intensity contrast of 38 . In the collinear setup, $V=22.9 \%$, or a contrast of 76 . In the non-collinear case, the fringe visibility significantly decreases to $5.1 \%$, or a contrast of $>1500$. Shown for reference is the transmission of a $300 \mathrm{~nm}$ thick zirconium filter, which has been used to compress attosecond pulses to a near transform limit $[8,36]$. By using a perturbing arm, we are able to improve the isolation of the attosecond pulses near the cutoff photon energy without significantly reducing the peak intensity.

An alternative approach to controlling the isolated portion of the spectrum is to manage the dispersion of the fundamental field. Through the spatio-temporal coupling of the fundamental field required for the attosecond lighthouse, the temporal character of the pulse also controls its spatial distribution. Before the focus, the coupled field spatial distribution is a combination of the pulse front tilt and the spatial chirp, resulting in the yaw. Anomalous dispersion results in a spatial chirp in the far field tilted pulse front. This far field spatial chirp compensates for the out-of-focus pulse front tilt, or the yaw. By minimizing the yaw, we optimize the wavefront curvature before the focus. In the case of $z=-z_{\mathrm{R}}$, shown in figure 4(a), the GDD of the fundamental field is -6 $\mathrm{fs}^{2}$. The resulting far field spectrum shows improved isolation above $75 \mathrm{eV}$ (b).

The anomalous dispersion required to optimize the curvature only slightly decreases the peak intensity, in spite of the initially short pulse duration used in the attosecond lighthouse. The spatial chirp increases the pulse duration by $\sqrt{2}$, or decreases the spatially-overlapped bandwidth at the focus by $\sqrt{2}$. Thus, the peak intensity is less sensitive to the imposed anomalous dispersion than the initially transform limited pulse.

\section{Results}

The experimentally measured spatially resolved XUV spectrum is shown in figure 5 . The experimental parameters are $I_{0}=5 \pm 0.5 \times 10^{14} \mathrm{~W} \quad \mathrm{~cm}^{-2}, \Delta t_{\mathrm{FWHM}}=4.5 \mathrm{fs}$, $\lambda \sim 760 \mathrm{~nm}$, and $w_{i}=2.5 \mathrm{~mm}$. The gas jet is neon with 4 Bar backing pressure and $250 \mu \mathrm{m}$ nozzle diameter; the gas jet 
position is $-z_{\mathrm{R}} / 4$. Without the perturbing field (a), the attosecond pulses overlap near the maximum photon energy, above $80 \mathrm{eV}$. The non-collinear perturbing field (b), $I_{p} / I_{0}=0.2 \%$ and angle $\theta=8 \mathrm{mrad}$, increases the separation of the attosecond pulses at the cutoff. (Note that the spot at $67 \mathrm{eV}, 2 \mathrm{mrad}$ is the decreased MCP response due to a previous experiment.)

The wedge insertion into the beam affects the GDD and the CEP of the fundamental field. The fused silica wedge has an apex angle of $8^{\circ}$ and is used to optimize the pulse compression. The resulting directed pulses are shown in figure 6 at $85 \pm 5 \mathrm{eV}$. The dispersion is assumed to be zero at the optimal separation of the low energy $(35 \mathrm{eV})$ portion of the spectrum. The optimum separation of the pulses at $85 \mathrm{eV}$ is at GDD $=-5 \mathrm{fs}^{2}$. The rapid slope is due to the CEP dependence of the direction of the generated attosecond pulses.

\section{Conclusion}

We have shown that the isolation of the pulses in the attosecond lighthouse is strongly dependent on the position of the gas jet relative to the focus. The necessary requirement for the isolation of the attosecond pulses in the far field is that their divergence (dependent on the focussing conditions) must be less than their separation (dependent on the wavefront rotation of the fundamental field). Moving the gas jet before the focus favours the isolation of the higher photon energy portion of the spectrum. However, the decrease in fundamental intensity limits the effectiveness of this technique. We find that using a perturbing field, in either collinear or non-collinear geometry, improves the isolation at the maximum photon energy without significantly sacrificing peak intensity. Alternatively, working in the anomalous dispersion regime can tune the isolated spectral region. This second approach is simple to implement and does not require additional (interferometrically stable) fields. These methods improve the contrast of the attosecond pulses generated from the attosecond lighthouse at the maximum photon energy. The high energy is necessary for implementing the attosecond lighthouse technique for water-window and soft $\mathrm{x}$-ray experiments [37, 38].

\section{Acknowledgments}

We thank Dr Andrei Naumov and Professor Paul Corkum for many useful discussions, and the technical assistance of David Crane and Bert Avery. TH acknowledges financial support from AFOSR MURI grant number FA9550-15-1-0037.

\section{References}

[1] Paul P M, Toma E S, Breger P, Mullot G, Augé F, Balcou Ph, Muller H G and Agostini P 2001 Science 292 1689-92
[2] Mairesse Y et al 2003 Science 302 1540-3

[3] Corkum P B 1993 Phys. Rev. Lett. 71 1994-7

[4] Corkum P B and Krausz F 2007 Nature Phys. 3 381-7

[5] Krausz F and Ivanov M 2009 Rev. Mod. Phys. 81 163-234

[6] Sola I J et al 2006 Nature Phys. 2 319-22

[7] Sansone G et al 2006 Science 314 443-6

[8] Goulielmakis E et al 2008 Science 3201614

[9] Wirth A et al 2011 Science 334 195-200

[10] Abel M J, Pfeifer T, Nagel P M, Boutu W, Bell M J, Steiner C P, Neumark D M and Leone S R 2009 Chem. Phys. 366 9-14

[11] Ferrari F, Calegari F, Lucchini M, Vozzi C, Stagira S, Sansone G and Nisoli M 2010 Nature Phot. 4 875-9

[12] Altucci C et al 2010 Opt. Lett. 35 2798-800

[13] Timmers H, Sabbar M, Hellwagner J, Kobayashi Y, Neumark D M and Leone S R 2016 Optica 3 707-10

[14] Oishi Y, Kaku M, Suda A, Kannar F and Midorikawa K 2006 Opt. Express 14 7230-7

[15] Mashiko H, Gilbertson S, Li C, Khan S D, Shakya M M, Moon E and Chang Z 2008 Phys. Rev. Lett. 100103906

[16] Feng X, Gilbertson S, Mashiko H, Wang H, Khan S D, Chini M, Wu Y, Zhao K and Chang Z 2009 Phys. Rev. Lett. 103183901

[17] Hammond T J, Kim K T, Zhang C, Villeneuve D M and Corkum P B 2015 Opt. Lett. 40 1768-70

[18] Hammond T J, Brown G G, Kim K T, Villeneuve D M and Corkum P B 2016 Nature Phot. 10 171-6

[19] Vincenti H and Quéré F 2012 Phys. Rev. Lett. 108113904

[20] Heyl C M, Bengtsson S N, Carlstrom S, Mauritsson J, Arnold C L and L'Huillier A 2014 New. J. Phys. 16052001

[21] Wheeler J A, Borot A, Monchocé S, Vincenti H, Ricci A, Malvache A, Lopez-Martens R and Quéré F 2012 Nature Phot. 6 829-33

[22] Kim K T, Zhang C, Ruchon T, Hergott J-F, Auguste T, Villeneuve D M, Corkum P B and Quéré F 2013 Nature Phot. 7 651-6

[23] Louisy M et al 2015 Optica 2 563-6

[24] Quéré F et al 2014 J. Phys. B: At Mol. Opt. Phys. 47124004

[25] Sansone G, Benedetti E, Vozzi C, Stagira S and Nisoli M 2008 New J. Phys. 10025006

[26] Marceau C, Gingras G and Witzel B 2011 Opt. Express 19 3576-91

[27] Akturk S, Gu X, Bowlan P and Trebino R 2010 J. Opt. 12 093001

[28] Lewenstein M, Balcou Ph, Ivanov M Yu, L'Huillier A and Corkum P B 1994 Phys. Rev. A 49 2117-32

[29] Lewenstein M, Salières P and L'Huillier A 1995 Phys. Rev. A 52 4747-54

[30] Balcou P, Dederichs A S, Gaarde M B and L'Huillier A 1999 J. Phys. B: A. Mol. Opt. Phys. 32 2973-89

[31] Gaarde M B, Tate J L and Schafer K J 2008 J. Phys. B: At. Mol. Opt. Phys. 41132001

[32] Balcou P, Salières P, L'Huillier A and Lewenstein M 1997 Phys. Rev. A 55 3204-10

[33] Auguste T, Gobert O, Ruchon T and Quéré F 2016 Phys. Rev. A 93033825

[34] Siegman A E 1986 Lasers (Sausalito, CA: University Science Books) pp 626-36

[35] Li Z, Brown G G and Corkum P B 2016 Proc. Canadian Association of Physicists Congress (https://indico.cern.ch/ event $/ 472838 /$ contributions $/ 1150181 /$ )

[36] Zhao K, Zhang Q, Chini M, Wu Y, Wang X and Chang Z 2012 Opt. Lett. 37 3891-3

[37] Zhang C, Vampa G, Villeneuve D M and Corkum P B 2015 J. Phys. B: At. Mol. Opt. Phys. 48061001

[38] Silva F, Teichmann S M, Cousin S L, Hemmer M and Biegert J 2015 Nature Comm. 66611 